each case) having no depression were selected as controls. The cases and controls were studied and matched for a myriad of sociodemographic factors. The various risk factors for depression were evaluated using univariate and multivariate binary logistics analysis.

Result. The significant risk factors for depression among hemodialysis patients were age $(\mathrm{OR}=1.79, \mathrm{CI}=0.47-3.81)$, comorbidities $(\mathrm{OR}=2.13, \mathrm{CI}=0.51-3.96)$, duration of renal disease $(\mathrm{OR}=2.54$, CI 0.63-4.28), duration of hemodialysis $(\mathrm{OR}=2.36, \mathrm{CI}=0.89$ 4.11), unemployment $(\mathrm{OR}=2.33, \mathrm{CI}=0.79-3.88)$, and being unmarried $(\mathrm{OR}=1.93, \mathrm{CI}=0.44-3.53)$. Prospect of survival, financial instability, social stigmatization, and effect of comorbidities on ESRD were major concerns for the cases that attributed to their depressive symptoms.

Conclusion. The factors that herald the onset of depression among hemodialysis patients include increasing age, presence of comorbidities, unemployment being unmarried, and increasing duration of hemodialysis. These factors will aid the clinicians to identify high-risk patients that require psychiatric consultation. We recommend prompt psychiatric intervention (pharmacologic or non-pharmacologic) and appropriate patient counseling so that the depressive symptoms can be alleviated and dismal disease prognosis can be prevented among such high-risk patients.

Lifetime depression and age-related changes in body composition, cardiovascular function, grip strength and lung function: sex-specific analyses in the UK Biobank

Julian Mutz ${ }^{1 *}$ and Cathryn M Lewis ${ }^{2}$

${ }^{1}$ Social, Genetic \& Developmental Psychiatry Centre, Institute of Psychiatry, Psychology \& Neuroscience, King's College London and ${ }^{2}$ Social, Genetic \& Developmental Psychiatry Centre, Institute of Psychiatry, Psychology \& Neuroscience, King's College London, Department of Medical and Molecular Genetics, Faculty of Life Sciences \& Medicine, King's College London

${ }^{\star}$ Corresponding author.

doi: 10.1192/bjo.2021.166

Aims. Individuals with mental disorders, on average, die prematurely, have higher levels of physical comorbidities and may experience accelerated ageing. In individuals with lifetime depression and healthy controls, we examined associations between age and multiple physiological measures.

Method. The UK Biobank study recruited $>500,000$ participants, aged 37-73 years, between 2006-2010. Generalised additive models were used to examine associations between age and grip strength, cardiovascular function, body composition, lung function and bone mineral density. Analyses were conducted separately in males and females with depression compared to healthy controls.

Result. Analytical samples included up to 342,393 adults (mean age $=55.87$ years; $52.61 \%$ females). We found statistically significant differences between individuals with depression and healthy controls for most physiological measures, with standardised mean differences between -0.145 and 0.156 . There was some evidence that age-related changes in body composition, cardiovascular function, lung function and heel bone mineral density followed different trajectories in individuals with depression. These differences did not uniformly narrow or widen with age. For example, BMI in female cases was $1.1 \mathrm{~kg} / \mathrm{m} 2$ higher at age 40 and this difference narrowed to $0.4 \mathrm{~kg} / \mathrm{m} 2$ at age 70 . In males, systolic blood pressure was $1 \mathrm{mmHg}$ lower in cases at age 45 and this difference widened to $2.5 \mathrm{mmHg}$ at age 65 .

Conclusion. Individuals with depression differed from healthy controls across a broad range of physiological measures.
Differences in ageing trajectories differed by sex and were not uniform across physiological measures, with evidence of both age-related narrowing and widening of case-control differences.

\section{Psychiatric presentations in acute illness with COVID-19: a retrospective analysis}

Elizabeth Nelmes ${ }^{\star}$ and Audrey Ng

West London NHS Trust

${ }^{*}$ Corresponding author.

doi: 10.1192/bjo.2021.168

Aims. To assess the psychiatric presentations in patients with a diagnosis of COVID-19 referred to a liaison psychiatry department during a one month period in the peak of the global pandemic.

Method. A retrospective analysis of the patients referred to liaison psychiatry during January 2021 who also had a diagnosis of COVID-19. Confirmed cases of COVID-19 were defined as those confirmed by COVID-19 PCR in respiratory samples or clinically suspected cases from chest radiograph or CT. Severe COVID-19 was defined as those requiring supplementary oxygen due to saturations of $93 \%$ or less.

Result. During January 2021, a total of 24 patients were referred to liaison psychiatry with concurrent COVID-19 infection. Out of these patients, $63 \%$ had a previous mental health diagnosis. The most common reason for referral was low mood (37.5\%), followed by agitation $(25 \%)$ and psychosis $(25 \%)$. When considering first psychiatric presentations with concurrent COVID-19 infection, the most common presentation was psychosis (44\%). The time course of psychosis was most frequently seen in the seven days prior to a positive swab. In one case a patient was sectioned under the Mental Health Act for psychosis two days prior to developing symptoms. Two of these patients were worked up for possible encephalitis including radiological imaging and lumbar puncture. For patients defined as having severe COVID-19, the most common referral was low mood. In those referred for low mood, $66 \%$ had a history of an affective disorder. In two cases low mood was complicated by an acute stress reaction to recent bereavement. For one patient this included the bereavement of two relatives to COVID-19. For patients admitted to intensive care and intubated for respiratory support the most common referrals were low mood and agitation. These factors we found a barrier to successful rehabilitation following periods of significant illness.

Conclusion. The impact of COVID-19 on psychiatric presentations extends beyond the socio-economic factors precipitating crises across the nation. Our findings of acute psychiatric illness in the prodromal phase of the viral illness suggest a neuropsychiatric pathogenesis to COVID-19.

Contribution of birth weight to mental health, cognitive, and socioeconomic outcomes: a two-sample Mendelian randomisation

Massimiliano Orri ${ }^{1 \star}$, Jean-Baptiste Pingault ${ }^{2}$, Gustavo Turecki ${ }^{3}$, Anne-Monique Nuyt ${ }^{4}$, Richard E Tremblay ${ }^{4}$, Sylvana M Côté ${ }^{4}$ and Marie-Claude Geoffroy ${ }^{3}$

${ }^{1}$ Bordeaux Population Health Research Centre, Mcgill University; ${ }^{2}$ University College London; ${ }^{3}$ Mcgill University and ${ }^{4}$ University of Montreal

${ }^{*}$ Corresponding author.

doi: 10.1192/bjo.2021.169 
Aims. Low birth weight is associated with adult mental health, cognitive, and socioeconomic problems. However, the causal nature of these associations remains difficult to establish due to confounding. We aimed to estimate the contribution of birth weight to adult mental health, cognitive, and socioeconomic outcomes using two-sample Mendelian randomisation, an instrumental variable approach strengthening causal inference.

Method. We used 48 independent single-nucleotide polymorphisms as genetic instruments for birth weight ( $\mathrm{N}$ of the genomewide association study, 264 498), and considered mental health (attention-deficit hyperactivity disorder [ADHD], autism spectrum disorders, bipolar disorder, major depressive disorders, obsessive-compulsive disorder, post-traumatic stress disorder [PTSD], schizophrenia, suicide attempt), cognitive (intelligence), and socioeconomic (educational attainment, income, social deprivation) outcomes. We performed a two-sample Mendelian randomisation using the random-effect Inverse Variance Weighing method as primary analysis, supplemented by a wide range of sensitivity analyses, including Egger regression, weighted median, and Pleiotropy Residual Sum and Outlier. Results were considered statistically significant after accounting for multiple testing using False Discovery Rate $(\mathrm{q}=0.05)$.

Result. After correction for multiple testing, we found evidence for a contribution of birth weight to ADHD (OR for 1 SD-unit decrease [ 464 grams] in birth weight, 1.29; CI, 1.03-1.62), PTSD $(\mathrm{OR}=1.69 ; \mathrm{CI}=1.06-2.71)$, and suicide attempt $(\mathrm{OR}=$ 1.39; $\mathrm{CI}=1.05-1.84)$, as well as for intelligence $(\beta=-0.07$; $\mathrm{CI}=$ $-0.13 ;-0.02)$, and socioeconomic outcomes, ie, educational attainment $(\beta=-0.05 ; \mathrm{CI}=-0.09 ;-0.01)$, income $(\beta=-0.08 ; \mathrm{CI}=$ $-0.15 ;-0.02)$, and social deprivation $(\beta=0.08 ; C I=0.03 ; 0.13)$. However, no evidence was found for a contribution of birth weight to the other examined mental health outcomes. Results were consistent across main and sensitivity analyses.

Conclusion. These findings support that birthweight could be an important element on the causal pathway to mental health, cognitive and socioeconomic outcomes. Early interventions targeting birth weight may therefore have a positive impact on promoting mental health and improving socioeconomic outcomes.

This project has received funding from the European Union's Horizon 2020 research and innovation programme under the Marie Skłodowska-Curie grant agreement No 793396

\section{Clinical audit of prescribing for attention deficit} hyperactivity disorder (ADHD) in children and young people services (CYPS)

Mary Parker* and Elaine Martin

Tees, Esk and Wear Valleys NHS Foundation Trust

${ }^{*}$ Corresponding author.

doi: 10.1192/bjo.2021.170

Background. The audit aimed to assess, when $3 \mathrm{rd}$ and 4 th line medications were prescribed for ADHD, if practice was compliant with Tees, Esk and Wear Valleys NHS Foundation Trust (TEWV) prescribing guidelines and the updated NICE Guideline NG87: Attention deficit hyperactivity disorder: diagnosis and management 2018.

Method. The audit was conducted in the four Teesside Child \& Adolescent community teams during April/May 2018. Each team identified all patients prescribed 3rd and 4th line ADHD medications leading to 30 responses $(n=30)$.

Information was collected from electronic and paper medical records using a designated audit tool compiled from the above evidence based guidelines. The data were analysed for compliance against standards using an excel spreadsheet and reviewed by the audit lead.

Result. There were many areas of good practice demonstrated in the audit including diagnostic recording, pre-treatment nonmedical interventions where ADHD was not severe, and use of Methylphenidate as first line medication in accordance with BNF limits. In the majority of records reviewed, there was good evidence of a variety of NICE recommended non-medication interventions which were often continued post medication initiation.

There was also very good evidence of comprehensive verbal and written information and psychoeducation including benefits and potential side effects of medication (92\% verbal and 58\% written).

A pre-treatment assessment was completed in all but 3 cases, 1 of which had no assessment documented and 2 cases were transferred from out of area.

Issues identified by the audit, where there was deviation from guidelines, included 4 cases where Methylphenidate was not prescribed as first line, of these, 3 were prescribed Atomoxetine due to parental choice and one was due to contraindications, suggesting patient choice was an important factor in selection of 2 nd line medication.

The audit demonstrates that clinical practice had moved away from the previous guidance in NICE CG72 (to prescribe atomoxetine 2nd line) towards the prescription of Lisdexamfetamine 2nd line (75\%) as reflected in the new NICE guidelines: NG87, 2018 (updated 2019).

Conclusion. This audit cycle has demonstrated that use of an evidence based approach has been instrumental in improving patient care. The Audit evidenced good practice in areas such as preassessment, information and psychological education, initial use of Methylphenidate, use of Lisdexamfetamine 2nd line, as well as consideration of patient choice. Importantly the audit highlighted that implementation of updated NICE compliant trust guidance, followed by a planned trust-wide audit will promote continuous improvement in patient care.

Pregnancy and contraceptive questioning within acute inpatient psychiatric admissions: are we asking enough?

\section{Eleanor Partington}

Newcastle University, Cumbria, Northumberland, Tyne And Wear NHS Foundation Trust

doi: 10.1192/bjo.2021.171

Aims. This audit explored how regularly women of childbearing age on an acute psychiatric inpatient ward are asked about pregnancy and contraception.

Background. Unplanned pregnancies and poor compliance with contraception are common in women with severe mental illness, with a significant number seeking abortions or losing custody of their children. As these women are also less likely to consult medical professionals, an admission is an essential point for intervention and support.

Additionally, there are risks associated with prescribing psychotropic medications during pregnancy. Because of this, The Royal College of Psychiatrists and local guidelines state that all female patients admitted onto psychiatric inpatient wards should be asked about their sexual health within seven days of admission.

Method. Data was collected from all 51 women of childbearing age admitted to a mixed-sex, acute adult inpatient psychiatry ward over one year, from January 2019 until the end of December 2019. 